\title{
Production of High Brightness Proton Bunches*
}

\author{
Thomas Roser \\ Brookhaven National Laboratory, Upton, NY 11973-5000, USA
}

\begin{abstract}
Strongly pulsed proton beams for secondary beam production are required for projects such as pulsed spallation neutron sources or neutrino factories where accurate timeof-flight information is required. To meet these demands techniques to produce multi-GeV proton bunches with very high longitudinal brightness are being developed. A review of the present status is presented
\end{abstract}

\section{INTRODUCTION}

The usefulness of secondary particle beams is greatly enhanced by strongly pulsing the high intensity proton beams that are typically used for the production process. With a well known production time one can either infer the secondary particle's energy from the elapsed time between detection and production, discriminate against out-of-time background events or produce secondary particle beams with a small longitudinal emittance.

An important example for the first type of application of pulsed particle production are the pulsed spallation neutron sources where the neutron time-of-flight information is used to select the neutron's energy. By increasing the bunching factor - the ratio of peak over average current - very low energy or cold neutrons can be clearly identified. The 2 MW Spallation Neutron Source (SNS), now under construction at Oak Ridge National Laboratory, will compare favorably with much higher power reactor-based neutron sources because of the pulsed nature if its neutron source.

Recently the $24 \mathrm{GeV}$ high intensity proton beam from the AGS was used to produce muons for the "muon g-2" experiment where the muon spin precessions are counted starting from the production time. In section 3 the AGS operations for this experiment will be presented and the main issues of high brightness proton bunch production discussed.

The suppression of background by using pulsed beams has been widely used with neutrino beams. Since neutrinos are practically massless the beam time structure will be preserved over large distances. The background suppression is then simply proportional to the bunching factor. This is being used at the $\mathrm{K} 2 \mathrm{~K}$ "long baseline" experiment where neutrinos produced at KEK with the $12 \mathrm{GeV}$ proton beam travel hundreds of miles before being detected at Super-Kamiokande.

Finally the production of low emittance secondary beams is important for secondary beam colliders. Antiproton beams have been used at the Spp̄S at CERN and

\footnotetext{
${ }^{*}$ Work performed under the auspices of the U.S. Department of Energy
}

at the FNAL Tevatron. However, the stable anti-protons allow for extensive stochastic cooling before the beams are used in the collider. This reduces the requirements on the initial secondary beam emittance.

This would be different for a muon collider. Ionization cooling is effective mostly in transverse phase space. Initial capture of the pion beam requires a longitudinal emittance that is as small as possible. Since the production process will produce a very large momentum spread the only way to reduce the secondary beam emittance is for the proton bunch length to be as short as possible.

The production of high brightness proton bunches requires both high intensity as well as small longitudinal emittance. A small transverse emittance is typically not required since the emittance of the secondary beam is dominated by the production process. In fact, the transverse emittance is often as large as possible to minimize space charge effects.

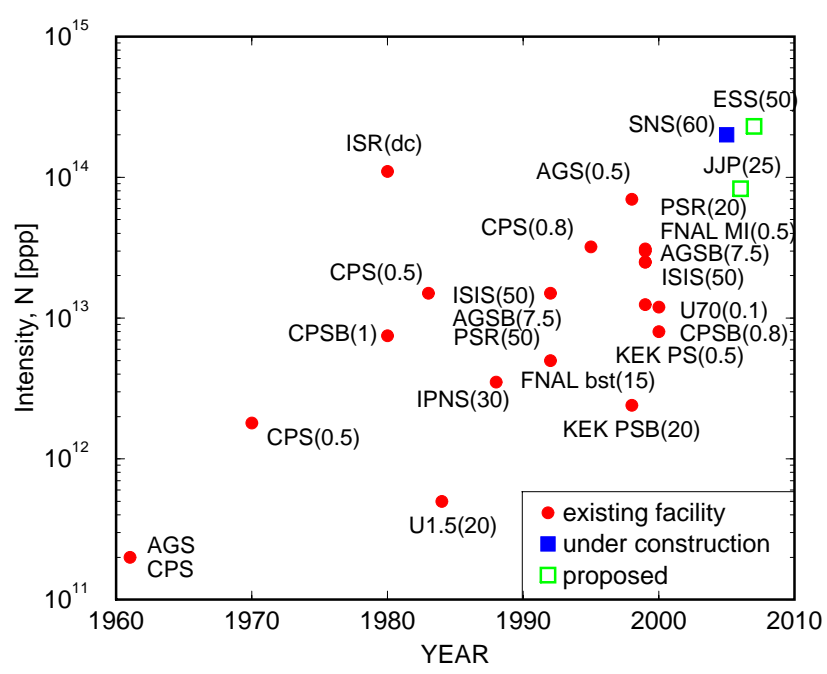

Figure 1: Intensity history of multi-GeV proton accelerators. The numbers in parenthesis indicate the typical repetition rate.

Fig. 1 shows a history of achieved proton intensity per accelerator pulse in multi-GeV machines[1]. As can be seen the progress has been exponential - very much like the famous Livingston plot of the peak beam energy. It is also interesting to note that the original alternating gradient proton accelerators - the CERN PS and the Brookhaven AGS - are still at the intensity frontier.

High proton pulse intensity by itself does imply high longitudinal brightness due to the finite circumference and momentum aperture of the accelerator. However, higher brightness can be achieved at lower intensity by avoiding dilution during injection, capture and acceleration. Ta- 
ble1 shows present and planned performance parameters of multi-GeV proton accelerators[2]. Also included are beam performance requirements for the injectors of high luminosity hadron colliders. In this latter case transverse brightness is, of course, also important.

Table 1: Longitudinal brightness of existing and planned proton machines

\begin{tabular}{|l|c|c|c|}
\hline & $\begin{array}{c}E_{\max } \\
{[\mathrm{GeV}]}\end{array}$ & $\begin{array}{c}N_{b} \\
{\left[10^{12}\right]}\end{array}$ & $\begin{array}{c}N_{b} / \epsilon_{L} \\
{\left[10^{12} / \mathrm{eVs}\right]}\end{array}$ \\
\hline Existing: & & & \\
FNAL Booster & 8 & 0.05 & 0.5 \\
DESY PETRA II & 40 & 0.08 & 0.7 \\
KEK PS & 12 & 0.9 & 1.0 \\
DESY III & 7.5 & 0.11 & 1.2 \\
FNAL MI & 150 & 0.12 & 1.2 \\
CERN PS & 14 & 1.25 & 1.8 \\
BNL Booster & 1.9 & 15 & 5.0 \\
BNL AGS & 24 & 12 & $1.2-4.0$ \\
LANL PSR & 0.8 & 23 & 18 \\
RAL ISIS & 0.8 & 12.5 & 21 \\
\hline Planned: & & & \\
P Driver Study I & 16 & 7.5 & 3.8 \\
P Driver Study II & 24 & 17 & 3.4 \\
Japan JHF & 50 & 12.5 & 2.5 \\
ORNL SNS & 1.0 & 200 & 20 \\
AGS for RHIC & 25 & 0.4 & 1.3 \\
PS for LHC & 26 & 0.9 & 0.9 \\
SPS for LHC & 450 & 0.1 & 0.2 \\
\hline
\end{tabular}

\section{LIMITATIONS TO PROTON BUNCH BRIGHTNESS}

Most high intensity proton machine presently use charge exchange injection of negatively charged hydrogen beams. The achievable brightness of the circulating beam can therefore be the brightness of the injected beam times the number of injected turns - a number that is between about 100 and 1000 . Whereas the transverse brightness is strongly limited by space charge driven tune spread the longitudinal brightness can indeed be very high.

The main limitations for the longitudinal brightness come from collective beam instabilities driven by coupling impedances or electron clouds that are captured by the potential well of the proton beam. The effect of electron clouds is most pronounced for coasting beams or long bunch trains that keep the electrons captured long enough for an instability to develop. In this sense an electron cloud driven instability does not limit, in principle, the production of high brightness proton bunches.

The coupling impedance contains a low frequency component from the resistive wall of the vacuum chamber and a high frequency broad band component $(Q \approx 1)$ from steps and bellows in the vacuum chamber. For the commonly observed "slow" head-tail instability the tail of the bunch can be driven resonantly by the wake field of the head for the correct chromatic betatron phase shift between the head and the tail. The growth rate is much smaller than the synchrotron frequency and is proportional to bunch intensity and chromaticity. Amplitude dependent tune shifts and also coupling[3] can provide an effective threshold for this instability.
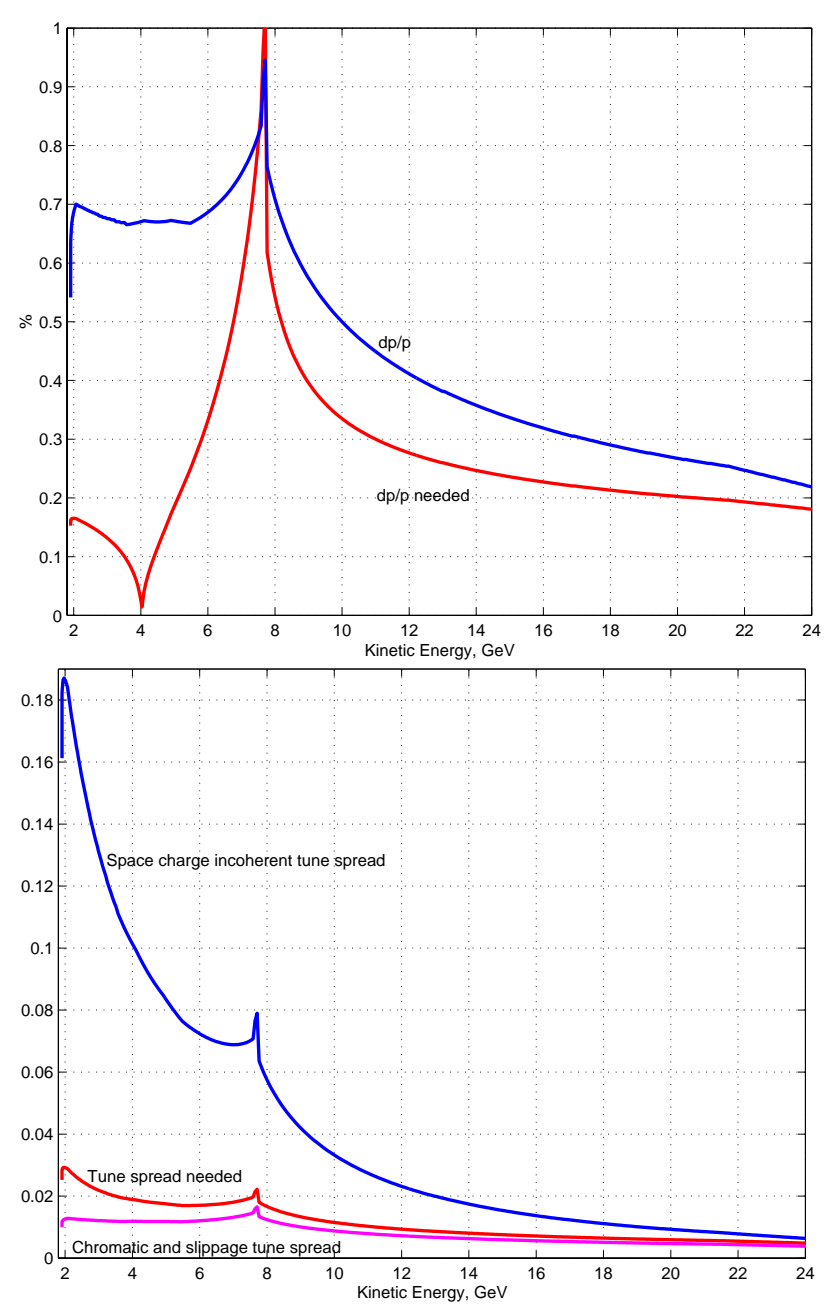

Figure 2: Stability threshold and actual momentum and tune spread for the longitudinal (top) and transverse (bottom) microwave instability for AGS high intensity operation with $1 \times 10^{13}$ protons per $6 \mathrm{eVs}$ bunch.

Of bigger concern are the longitudinal and transverse microwave instabilities. In this case the tune shifts induced by the coupling impedance are bigger than the synchrotron tune leading to possibly very fast instability growth.

Unlike electron beams proton bunches are typically much longer than the vacuum chamber diameter. For these fast instabilities the threshold is then very similar to threshold for a coasting beam[5] with the average beam current replaced with the peak current of the bunch.

For the longitudinal instability the minimum stable beam 
momentum spread is then given by

$$
\left(\frac{\Delta p}{p}\right)^{2}>\frac{e I_{p e a k}}{E|\eta|}\left|\frac{Z_{L}(n)}{n}\right|
$$

The top part Fig. 2 shows the threshold and beam momentum spread for high intensity AGS operation. The longitudinal impedance includes both the $30 \Omega$ broad band and the space charge impedance. Longitudinal space charge impedance at low energy could be compensated by inductive inserts[4]. However, this instability is mostly of concern only around transition energy. Using a transition energy jump or - even better - avoiding transition crossing altogether avoids emittance growth from this longitudinal instability.

The transverse microwave instability often limits the single bunch intensity in electron machines. On would expect this instability to be also very important in high intensity proton machines because the coherent space charge tune shifts are very large and the synchrotron tune is typically much smaller than in electron machines. However, this instability has not yet been clearly identified in any proton machine. Whether this is simply due to the typically lower peak currents in proton machines or because of an additional stabilizing effect in proton machines has been the focus of extensive discussions. The effect of space charge on the transverse microwave instability has been studied by several people and typically space charge effects tend to stabilize the beam $[6,7,8]$.

The minimum tune spread for a transverse impedance $Z_{T}$ is given by

$$
\Delta \nu>\frac{e I_{\text {peak }} R}{4 \pi E \nu_{\beta}}\left|Z_{T}\right|
$$

The bottom part of Fig. 2 shows the threshold and beam tune spreads for high intensity AGS operation. The transverse impedance includes both the $3 \mathrm{M} \Omega / \mathrm{m}$ broad band and the coherent space charge impedance. It seems that the incoherent space charge tune spread is required for stability at injection energy[6]. There is also evidence that for very bright bunches in the AGS individual bunches sometimes "disappear" at the end of the acceleration cycle. This would be consistent with loosing stability as the space charge tune spread becomes smaller at higher energy although no direct observation of the instability could be made yet.

\section{HIGH BRIGHTNESS BEAM PRODUCTION AT THE AGS}

Fig. 3 shows the present layout of the AGS-RHIC accelerator complex. The high intensity proton beam of the AGS is used both for the slow-extracted-beam (SEB) area with many target station to produce secondary beams and the fast-extracted-beam (FEB) line used for the production of muons for the g-2 experiment and for high intensity target testing for the spallation neutron sources and muon production targets for the muon collider.

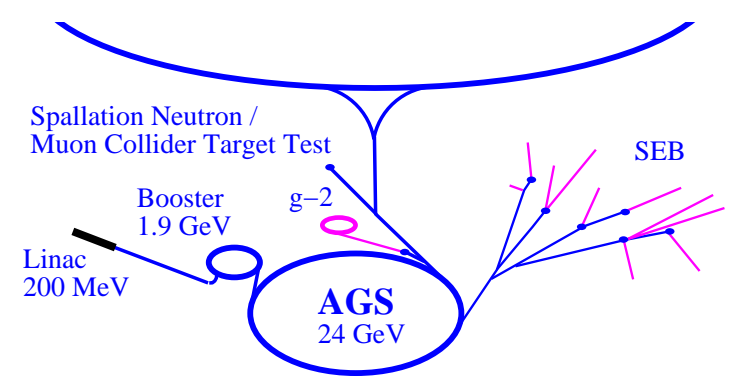

Figure 3: The AGS-RHIC accelerator complex.

The proton beam intensity in the AGS has increased steadily over the 40 year existence of the AGS, but the most dramatic increase occurred over the last few years with the addition of the new AGS Booster. The AGS Booster has one quarter the circumference of the AGS and therefore allows four Booster beam pulses to be stacked in the AGS at an injection energy of $1.5-1.9 \mathrm{GeV}$. At this increased energy, space charge forces are much reduced and this in turn allows for the dramatic increase in the AGS beam intensity.

\section{Injectors}

A recent upgrade of the $200 \mathrm{MeV}$ LINAC rf system made it possible to operated at an average $\mathrm{H}^{-}$current of $150 \mu A$ and a maximum of $12 \times 10^{13} \mathrm{H}^{-}$per $500 \mu \mathrm{s}$ LINAC pulse. Typical beam currents are $37 \mathrm{~mA}$ at end of the LINAC. The normalized beam emittance is about $10 \pi \mathrm{mm} \mathrm{mrad}$ for $95 \%$ of the beam and the beam energy spread is about $\pm 1.2 \mathrm{MeV}$.

The achieved beam intensity in the Booster reached a peak value of $2.3 \times 10^{13}$ protons per pulse. This was achieved by very carefully correcting all the important nonlinear orbit resonances especially at the injection energy of $200 \mathrm{MeV}$ and by using a second set of rf cavities as a second harmonic rf system. The typical bunch area of one of the two bunches was about $1.5 \mathrm{eVs}$. Even with the second harmonic rf system the incoherent space charge tune shift can reach one unit right at injection $\left(2.5 \times 10^{13}\right.$ protons, norm. $95 \%$ emittance: $50 \pi \mathrm{mm} \mathrm{mrad}$, bunching factor: 0.5). Of course, such a large tune shift is not sustainable, but the beam emittance growth and beam loss can be minimized by accelerating rapidly during and after injection. Best conditions are achieved by ramping the main field during injection with $3 \mathrm{~T} / \mathrm{s}$ increasing to $9 \mathrm{~T} / \mathrm{s}$ after about $10 \mathrm{~ms}$.

Recently the Booster rf system was modified to allow for the acceleration of a single bunch. At this lower frequency $45 \mathrm{kV}$ was available for the first harmonic and $22 \mathrm{kV}$ for the second harmonic. With only one bunch in the Booster a peak intensity of about $1.8 \times 10^{13}$ was reached with a bunch area of about $3 \mathrm{eVs}$. Single bunch operation in the Booster allowed for the transfer of six Booster loads into the AGS reducing the need for very high intensity in the Booster. 


\section{Acceleration in AGS}

The AGS itself also had to be upgraded to be able to cope with the higher beam intensity. Fig. 4 shows the total current and peak current in the AGS during operation for the g-2 experiment with 6 injections from the Booster and, after bunch splitting, 12 single bunch extraction to the g-2 production target.

During beam injection from the Booster, which cycles with a repetition rate of about $7 \mathrm{~Hz}$, the AGS needs to store the already transferred beam bunches for about 0.8 seconds. During this time the beam is exposed to the strong image forces from the vacuum chamber which will drive transverse beam instabilities. A very powerful transverse feedback system was installed to damp rigid bunch instabilities.

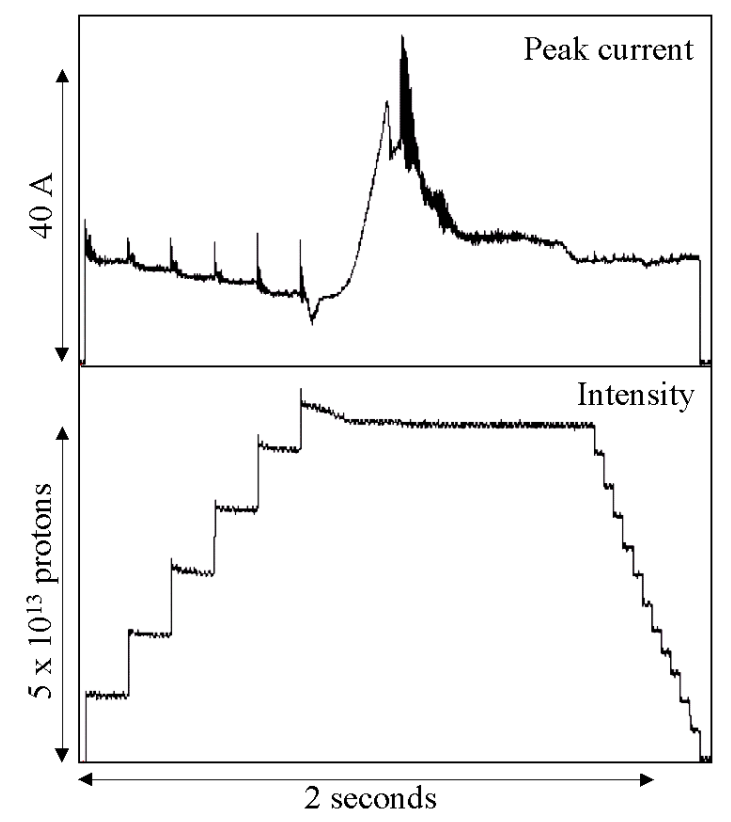

Figure 4: Total intensity and peak current in the AGS during operation for the $\mathrm{g}-2$ experiment.

The typical betatron tune setting is about 8.9 which is required to avoid the non-linear octupole stopband resonance at 8.75. With an incoherent tune shift at the AGS injection energy of 0.1 to 0.2 it is still necessary, however, to correct the octupole stopband resonances to avoid excessive beam loss.

To reduce the space charge forces further the beam bunches in the AGS are lengthened by purposely mismatching the bunch-to-bucket transfer from the Booster and then smooth the bunch distribution using a high frequency $100 \mathrm{MHz}$ dilution cavity. The resulting reduction of the peak current helps both with coupled bunch instabilities and stopband beam losses.

A large part of the injection losses at the AGS are due to a relative slow loss during the first millisecond the transferred bunches circulate in the AGS. No direct cause for this loss could be identified but it is correlated with a sus-

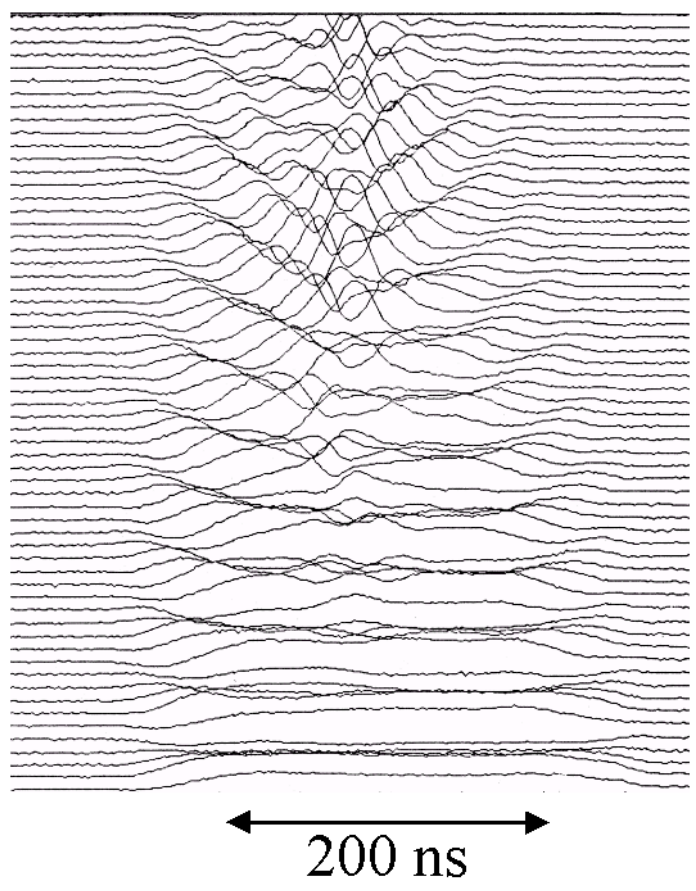

Figure 5: Evolution of the vertical miss-match at AGS injection. The traces show the vertical displacement of the same bunch every revolution. The horizontal scale is in microseconds

tained transverse coherent beam oscillation shown in Fig.5. The coherent oscillations result from miss-matched beam injection to blow-up the transverse emittance and therefore reduce the space charge tune shift. Although the coherence persists over a whole millisecond the middle part of the beam bunch has a coherent space charge tune shift of about 0.1 and therefore very high frequency vertical modulations appear. The bunch intensity is about $1.3 \times 10^{13}$ protons. The variation of the coherent space charge tune shift along the bunch could add to Landau damping of the transverse microwave instability[9].

At bunch intensities above $1.3 \times 10^{13}$ protons a vertical head-tail instability develops with a single bunch in the AGS. Fig. 6 shows both the bunch shape as well as the vertical modulation. The growth rate of about $50 \mathrm{~ms}$ is quite slow consistent with a weak head-tail instability. Only the middle part of the bunch was actually unstable indicating that the space charge tune depression of about 0.1 between the middle and the edge of the bunch can provide Landau damping. The observed asymmetry suggests that this instability is driven by the broad band impedance. The instability can easily be avoided by adjusting the chromaticity, coupling or betatron tunes.

During acceleration the AGS beam has to pass through the transition energy using a transition energy jump system with only minimal losses even at the highest intensities. After the transition energy, a very rapid, high frequency instability develops which could only be avoided by purposely further increasing the bunch area using again the high fre- 


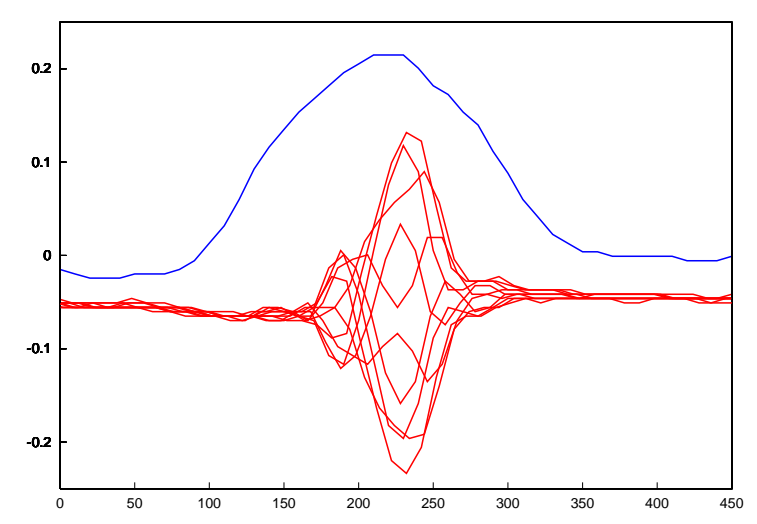

Figure 6: Bunch shape (top) and vertical modulation of a high intensity bunch at AGS injection energy of $1.9 \mathrm{GeV}$ during a single bunch head-tail instability. The vertical scale is in arbitrary units and the horizontal scale is in ns.

quency dilution cavity.

The peak beam intensity reached at the AGS extraction energy of $24 \mathrm{GeV}$ was $7.2 \times 10^{13}$ protons per pulse also exceeding the design goal for this latest round of intensity upgrades. It also represents a world record beam intensity for a proton synchrotron. Individual bunches with an intensity of $1.2 \times 10^{13}$ had a bunch area of about $3 \mathrm{eVs}$ at AGS injection but were diluted to about $10 \mathrm{eVs}$ during acceleration.

\section{Bunch compression}

During fast-extracted beam operation single bunches are extracted multiple times from the AGS at $24 \mathrm{GeV}$. The bunch length at extraction has to be as short as possible. This requirement conflicts with the effort to dilute the beam to stabilize it at high intensity. Various non-adiabatic methods to compress the bunch could be used. One is to lower the rf voltage adiabatically and then raising it quickly to get bunch compression after a quarter synchrotron period. Alternatively, the bunch could be moved onto the unstable fixpoint where it would be stretched out. After return to the stable fixpoint it compresses after some synchrotron rotation. Both methods give similar compression factors[10] limited mainly by non-linearities of the synchrotron motion. However, multiple single bunch extraction requires a reversible compression method. A technique to shorten the bunches before extraction was developed using adiabatic quadrupole pumping by modulating the rf voltage with a frequency close to twice the synchrotron frequency. With this technique a coherent quadrupole excitation as shown in Fig. 7 could be maintained for the multiple extractions[11]. This technique is also less affected by the non-linearities and could therefore reach higher compression factors.

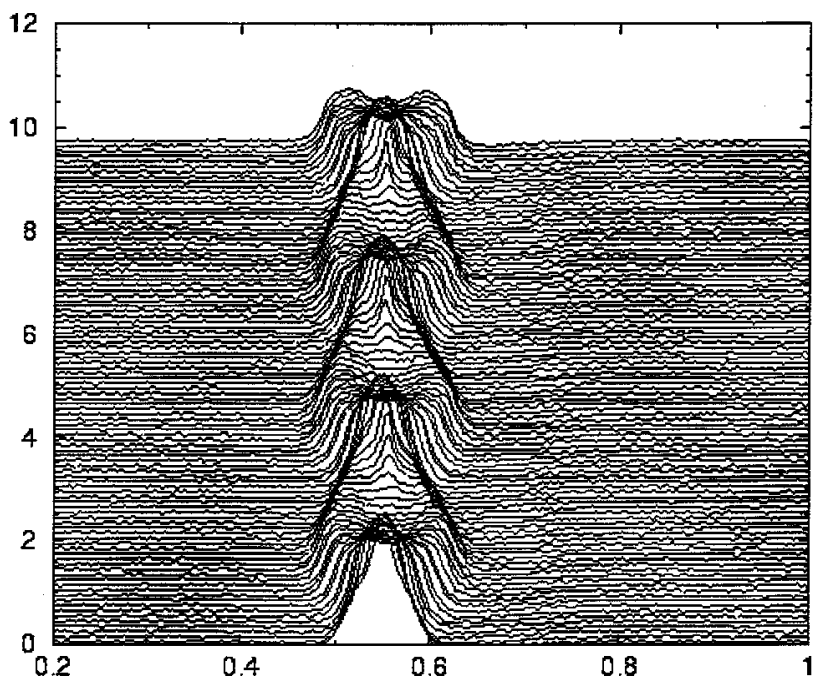

Figure 7: Evolution of the shape of a high intensity proton bunch during adiabatic quadrupole excitation.

\section{ACKNOWLEDGEMENT}

I would like to thank S.Y. Zhang and M. Blaskiewicz for many stimulating discussions.

\section{REFERENCES}

[1] J.Wei, A. Fedotov, and Y. Papaphilippou, "Physics and design of High intensity Circular Accelerators", Lecture at the 2001 U.S. Part. Accel. School.

[2] P. Martin and W. Chou, FNAL report FERMILAB-TM-1979 (1996); Neutrino Factory Feasibility Study, edited by N. Holtkamp and D. Finley (2000).

[3] R. Cappi, R. Garoby, E. Metral, Proceedings of Workshop on Instabilities of High Intensity Hadron Beams in Rings, edited by T. Roser and S.Y. Zhang, AIP Proceedings 496, (1999) 116.

[4] M. A. Plum et al., Phys. Rev. ST Accel. Beams 2, 064201 (1999).

[5] E. Keil and W. Schnell, CERN Report TH-RF/69-48 (1969); V.K. Neil and A.M. Sessler, Rev. Sci. Instr. 36 (1965) 429.

[6] S. Y. Zhang and W. T. Weng, 1997 Particle Accel. Conf., (1998) 1885

[7] M. Blaskiewicz, Phys. Rev. ST Accel. Beams 1, 044201 (1998).

[8] K.Y. Ng and A.V. Burov, Proceedings of Workshop on Instabilities of High Intensity Hadron Beams in Rings, edited by T. Roser and S.Y. Zhang, AIP Proceedings 496, (1999) 49.

[9] M. Blaskiewicz and W.T. Weng, Phys. Rev. E50, 4030 (1994)

[10] K.M. Fung et al., Phys. Rev. ST Accel. Beams 3, 100101 (2000).

[11] M. Bai et al., Phys. Rev. ST Accel. Beams 3, 064001 (2000).

[12] C. Ankenbrandt et al., Phys. Rev. ST Accel. Beams 1, 030101 (1998). 